\title{
X-RAY EVIDENCE FOR THE ACCELERATION, CONTAINMENT, AND EMISSION OF HIGH ENERGY FLARE PARTICLES
}

\author{
KENNETH J. FROST \\ Solar Plasmas Branch, Laboratory for Solar Physics, NASA-Goddard Space Flight Center, \\ Greenbelt, Md. 20771, U.S.A.
}

\begin{abstract}
An instrument aboard the Fifth Orbiting Solar Observatory has observed hard solar X-rays from January 1969 to May 1972. A large number of X-ray bursts generated by solar cosmic ray flares have been observed. The X-ray bursts consist, in general, of two non-thermal components. The earliest occurring non-thermal component, coincident with the explosive phase, consists of a group of one to about ten $\mathrm{X}$-ray bursts that are, for each burst, approximately $10 \mathrm{~s}$ duration and symmetrical in rise and decay. The time structure and multiplicity of these bursts is remarkably similar to that found in type III radio bursts in the meterwave band. The spectra of these bursts steepens sharply at energies greater than $100 \mathrm{keV}$ indicating a limit at this energy for electron acceleration during the explosive or flash phase of the flare. For several flares these multiple X-ray bursts have occurred in coincidence with a group of type III bursts.

The second non-thermal X-ray component usually begins almost immediately after the first and in the same time interval during which type II and type IV radio emission is detected. For several events there is a remarkable similarity between the time profile of the type IV burst and the X-ray burst. The rise time of this component is of the order of a minute or two but the decay time is of the order of tens of minutes. The spectral character is power law over the whole 14 to $250 \mathrm{keV}$ range. The index of the power varies from -6 to -2 and seems to track the hardness of the interplanetary solar electron spectral index.

We interpret these data as evidence that acceleration of solar particles occurs in two stages and that the X-ray components are the equivalents in the X-ray range of type III and type IV bursts in the radio range.
\end{abstract}

\section{DISCUSSION}

Smith: Has anyone tried to check the consistency of the hypothesis that the X-ray burst is associated with a moving type IV burst using the radio data? If so, what density is required?

Frost: We have not yet made a quantitative check on this speculation.

Dulk: The number of electrons in a plasmoid needed to get the radio emission is $10^{33}$ electrons with energy greater than $10 \mathrm{keV}$. There are about $10^{26}$ to $10^{27}$ erg involved in these electrons.

Frost: That number may be compatible with the number needed to explain the $\mathrm{X}$-ray burst.

Smerd: On the evidence presented by Mr Frost it seems feasible that those hard X-ray bursts which he tentatively associated with subsequent moving type IV bursts (at metre wavelengths) may be associated with those early, concurrent stationary type IV bursts at metre wavelengths which are referred to as flare continua. The latter occur characteristically at the time of the microwave type IV burst. 
Note added after discussion. The sources of flare continua are high in the corona, those of the microwave IV are low; it may require position determination of hard X-ray sources to determine whether they are radiated from near the microwave source, or the stationary or moving metre-wave source.

Kundu: I am glad that Dr Smerd mentioned that the $80 \mathrm{MHz}$ type IV source of March 21, 1969 was stationary and not moving as referred to by Mr Frost. It fits in nicely with the well known association of hard X-ray bursts with centimetre-wave bursts. This phase of metre-wave type IV was referred to by us as type IVA, which we interpreted as being due to the same source as centrimetre type IV. Consequently, I suggest that you should not rule out the possibility that the centimetre-continuum burst is as closely associated as the metre-wave type IV.

Regarding the lack of exact correspondence of the time profile of the hard X-ray burst with the centimetre burst, I would like to point out that the centimetre burst decreases in intensity and temperature during the post-burst phase; probably a better fit could be obtained if you compared it with another part of the X-ray spectrum. Further, the profile of a centimetre burst takes different shapes depending on the angular resolution of the beam used to track the region which flares up. In support of my contention, I'd like to show an interferometric record of a $3.7 \mathrm{~cm}$ burst of gradual rise and fall (g.r.f.) type, as obtained on June 9, 1973 with 3" resolution. The slide shows fringe amplitude as a function of time; the burst started around 1806 UT, had its maximum around 1816 UT and continued beyond 1830 UT. The g.r.f. had the same time of start and maximum. However, unlike the g.r.f. which is obtained with beams covering the entire Sun, the interferometric profile reveals the impulsive nature of the burst around its peak. A significant fraction of the burst energy is emitted within a spherical volume of diameter 2", with a corresponding brightness temperature of about $10^{9} \mathrm{~K}$.

Sturrock: If the moving type IV burst plasmoid produces X-rays it presumably contains a substantial mass of cool gas. Would you agree?

Frost: I intuitively expect the gas to be hot. 